\title{
Borrelia burgdorferi bacteremia
}

\section{Ruef}

Published online: 11 February 2011

(C) Urban \& Vogel 2011

Infections caused by Borrelia burgdorferi and other Borrelia species affect several organs, namely, the skin, heart, joints, and nervous system. The portal of entry is the skin following a tick bite. In order to cause infection in the other organs mentioned above, $B$. burgdorferi must gain access to the blood stream and reach these organs through the hematogenous route.

The detection of B. burgdorferi bacteremia causes some methodological problems, which may result in an underestimation of the frequency of bacteremia caused by these bacteria. B. burgdorferi will not grow in blood culture media, which are used in clinical routine practice. The study by Maraspin et al. [1] in this issue of INFECTION provides some interesting insights into the topic of B. burgdorferi bacteremia. The authors looked at the medical records of patients diagnosed with various forms of B. burgdorferi infections. The authors found that the presence of bacteremia is infrequent in patients with Lyme borreliosis other than erythema migrans. If erythema migrans is present, the isolation rate is in excess of $36 \%$. Interestingly, positive blood cultures were also found in patients with acrodermatitis chronica atrophicans of rather long duration.
These findings raise interesting questions regarding the usefulness of blood cultures using suitable media for the assessment of disease activity in various stages of the infection and in infections affecting various organs. Additional studies are certainly necessary to determine the clinical significance of bacteremia by B. burgdorferi. The sensitivity of the culture method would also need to be determined in a prospective study.

Although this study raises more questions than it answers, it provides interesting data, which might trigger future research activities on the pathogenesis of B. burgdorferi infections.

C. Ruef, Editor-in-Chief, INFECTION

\section{Reference}

1. Maraspin V, Ogrinc K, Ružić-Sabljić E, Lotrič-Furlan S, Strle F. Isolation of Borrelia burgdorferi sensu lato from blood of adult patients with borrelial lymphocytoma, Lyme neuroborreliosis, Lyme arthritis and acrodermatitis chronica atrophicans. Infection 2010 Dec 10 [Epub ahead of print]. Infection 2011; 39.
C. Ruef $(\square)$

University Hospital of Zurich, Zurich,

Switzerland

e-mail: christian.ruef@usz.ch 